\title{
Nanosheet transfection: effective transfer of naked DNA on silica glass
}

\begin{abstract}
Nien-Chi Huang ${ }^{1,5}$, Qingmin $\mathrm{Ji}^{2,5}$, Katsuhiko Ariga ${ }^{2,3}$ and Shan-hui Hsu ${ }^{1,4}$
Existing gene delivery technologies using nonviral vectors or physical stimulation are limited by cytotoxicity. Here, we reveal an easy and new method to fabricate silica upright nanosheets, which can be easily scaled up and used for gene delivery. We demonstrate that naked DNA can be transferred into difficult-to-transfect cells (for example, stem cells) by plating cells on silica glass with the upright nanosheets without using any vector. Gene entry is probably achieved through the integrin-FAK-Rho signaling axis, which can be activated by the cytoskeleton rearrangement on nanosheets in a limited time frame ('transfection window'). Transfecting naked GATA4-binding protein 4 plasmids into stem cells can upregulate the other two important cardiac marker genes myocyte enhancer factor $2 \mathrm{C}$ and T-box 5 . The transfected mesenchymal stem cells express the cardiac marker proteins at 7 days after transfection, which confirms the success of the innovative gene delivery approach. The vector-free silica nanosheet-induced transfection is simple and effective but faster and safer than the conventional technologies.
\end{abstract}

NPG Asia Materials (2015) 7, e184; doi:10.1038/am.2015.43; published online 5 June 2015

\section{INTRODUCTION}

Naked biomolecules, such as DNA, enter cells rather poorly. Thus, a therapeutic gene is often integrated with a viral or nonviral vector for intracellular delivery. Viral vectors have immune responses and safety concerns, whereas nonviral gene vectors or transfection reagents, such as cationic lipids, dendrimers and nanoparticles (NPs), may suffer from cytotoxicity. ${ }^{1}$ The NPs with positive charge bind to the gelled areas of the cell membrane to induce fluidity for particle penetration and disrupt the intracellular microtubule assembly. ${ }^{2,3}$ In addition to the vector-based delivery, a few physical or mechanical methods (for example, microinjection, gene gun, electroporation, sonoporation and laser irradiation) are used to deliver naked plasmid DNA, but they can also cause cell damage. ${ }^{4}$ It remains very challenging to deliver naked plasmid DNA without any vector or physical stimulation into normal cells and stem cells, which are difficult to transfect.

Intracellular gene delivery using a transfection reagent or physical method commonly involves forward transfection, where cells are seeded before exposure to the plasmid DNA. Meanwhile, reverse transfection is often associated with the substrate-mediated gene delivery, where plasmid DNA/transfection reagent complexes are pre-adsorbed on the culture substrate before cell seeding. ${ }^{5}$ The substrate that tethers more plasmid complexes, for example, substrates with nanostructures, can enhance the efficiency of gene transfection. ${ }^{6}$ Poly(methyl methacrylate) nanopillars were reported to improve the transfection of plasmid DNA/transfection reagent complexes into mesenchymal stem cells (MSCs). ${ }^{7}$ Meanwhile, silicon nanowires can pierce through the cell membrane and promote the transfection of plasmid DNA/transfection reagent complexes that are tethered on the nanowires. ${ }^{8}$ Coating the nanowires with aminosilane further improves the gene and protein delivery. ${ }^{9}$ In the existing technologies that involve either forward or reverse transfection, the efficiency of transfecting naked plasmid DNA without physically penetrating a cell remains notably low.

Cell-nanotopography interaction is known to alter cell behaviors. ${ }^{10,11}$ At the cell-material interface, the cell-membrane receptor 'integrins' have intricate roles in cell-material adhesion (focal adhesion, or FA), through which regulatory signals are transmitted. FA links the extracellular matrix to induce cytoskeleton contraction. ${ }^{12}$ The mechanical signals from extracellular matrix are conveyed to the nucleus by integrins and FA kinase (FAK) through the cytoskeleton. ${ }^{13}$ Nanogratings with 250-nm line width affect the expression of the integrin-activated FAK and cytoskeleton. ${ }^{14}$ Nanopost or nanograte structures induce FA formation and cytoskeleton changes. ${ }^{15}$ Nanopits reduce cell adhesion but increase the expression of an endocytosisassociated protein. ${ }^{16}$

Our previous study demonstrated that a dense and hydrophilic silica upright nanosheet network was conveniently fabricated by immersing silica-coated silicon in $\mathrm{NaBH}_{4}$ solution and heating. When modified

${ }^{1}$ Institute of Polymer Science and Engineering, National Taiwan University, Taipei, Taiwan, R.O.C; ${ }^{2}$ World Premier International Research Center for Materials Nanoarchitectonics, National Institute for Materials Science, Tsukuba, Japan; ${ }^{3}$ Japan Science and Technology Agency, Core Research for Evolutionary Science and Technology, Tsukuba, Japan and ${ }^{4}$ Research and Development Center for Medical Devices, National Taiwan University, Taipei, Taiwan, R.O.C

${ }^{5}$ These authors contributed equally to this work.

Correspondence: Professor S-h Hsu, Institute of Polymer Science and Engineering, National Taiwan University, No. 1, Sec. 4 Roosevelt Road, Taipei 10617, Taiwan, R.O.C. E-mail: shhsu@ntu.edu.tw

or Professor K Ariga, World Premier International (WPI) Research Center for Materials Nanoarchitectonics (MANA), National Institute for Materials Science (NIMS), 1-1 Namiki, Tsukuba 305-0044, Japan.

E-mail: ARIGA.Katsuhiko@nims.go.jp

Received 22 December 2014; revised 26 February 2015; accepted 27 March 2015 
a

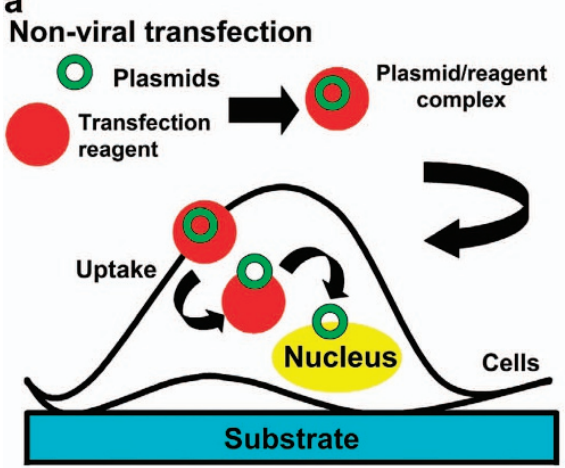

c

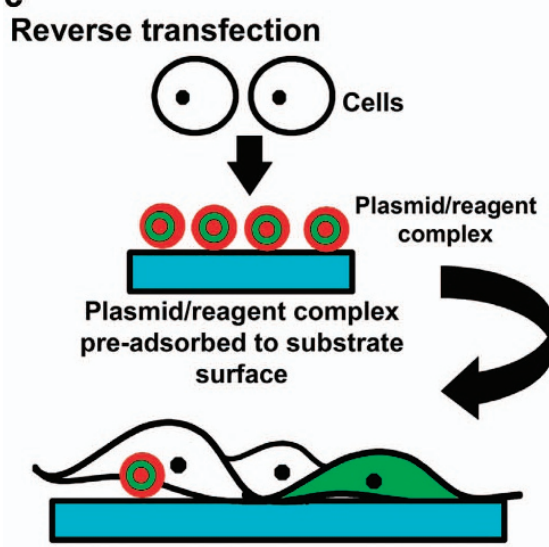

b

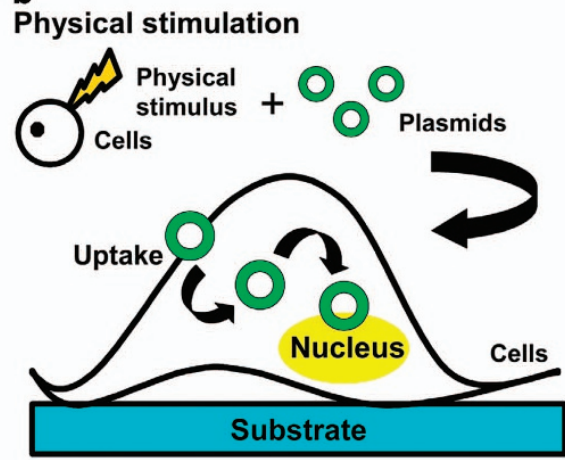

d

"Nano substrate"-induced gene delivery

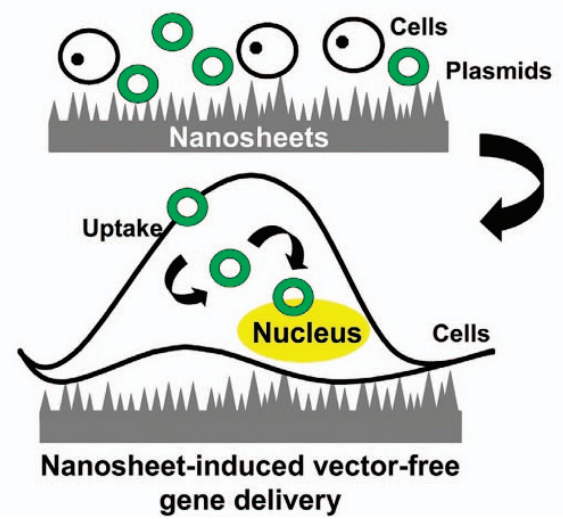

Figure 1 Schematics of common strategies (a-c) for gene delivery: (a) nonviral transfection, (b) physical or mechanical transfection, (c) substrate-mediated reverse transfection and (d) the new 'nanosubstrate'-induced naked gene delivery.

by 3-aminopropyltriethoxysilane, the material could enhance the transfer of a green fluorescent protein (GFP) reporter gene into human embryonic kidney (HEK) cells using the conventional vectorbased reverse transfection protocol. ${ }^{17}$ Because cell behavior and endocytosis are both affected by the integrin-nanotopography interaction, ${ }^{7,10}$ we assume that these upright nanosheets may also mediate the endocytosis and transfection of naked plasmid DNA. In this study, we reveal a new method that can be easily scaled up to produce silica upright nanosheets for practical use and a new method to transfer a therapeutic gene into difficult-to-transfect cells (for example, stem cells) using the silica upright nanosheets without any vector. The innovative technology is distinct from all existing gene delivery technologies, as shown in Figure 1. The transfection materials are abundant (silica glass) and prepared from simple wet etching. We confirm the intracellular delivery of naked plasmid on the silica upright nanosheets and suggest that the integrins/FAK/cytoskeleton regulation is the mechanism behind the vector-free gene delivery.

\section{MATERIALS AND METHODS}

The nanosheets with a featured wall depth of 5-nm covering planar glass were prepared using the following simple process. Silica was sputtered onto $1 \mathrm{~cm}^{2}$ silicon with a $10-\mu \mathrm{m}$-thick layer. The silicon was incubated and etched in $0.5 \mathrm{mg} \mathrm{ml}^{-1} \mathrm{NaBH}_{4}$ solution at $75^{\circ} \mathrm{C}$ for $6 \mathrm{~h}$ to form the suspended upright nanosheet network. After the reaction, the suspended nanosheets were harvested by centrifugation, washed and resuspended in clean water. Then, the nanosheet suspension $(600 \mu \mathrm{l})$ was dripped onto 1.5 -cm-diameter coverslip glass placed in a Petri dish and dried for $24 \mathrm{~h}$. This simple fabrication method successfully increases the yield of nanosheets compared with the previous study ${ }^{15}$ and is easier to scale up to adopt the technologies to gene delivery. The preparation process of the silica upright nanosheet network is shown in Figure 2a. The nanosheets can be directly used or modified with 3-aminopropyltriethoxysilane (ATPES) to increase the encounter of naked plasmid with cells through electrostatic interaction. In the latter, the upright nanosheet surface was aminosilylated with 5\% ATPES in water for $12 \mathrm{~h}$, washed with ethanol and water and dried at room temperature. Different amounts of etching time yielded upright nanosheets with various heights and thicknesses. The aforementioned etching time has been optimized to create upright nanosheets that are suitable for transfection. The surface morphology was examined using scanning electron microscopy. The surface hydrophilicity was evaluated by measuring the contact angle (FTA-1000B, First Ten Angstrom Company, Portsmouth, VA, USA). The surface zeta potential was determined using electrophoretic light scattering (Delsa Nano C, Beckman Coulter, Brea, CA, USA). The surface chemistry was characterized with attenuated total reflectance-infrared spectroscopy and an energy dispersive X-ray spectrometer. Surface functionalization by amino groups was also quantified via the reaction with 4-nitrobenzaldehyde (NBZ). ATPES-coated silica nanosheets were immersed in $10 \mathrm{ml}$ of dehydrated methanol, which contained $5 \mathrm{mg}$ of NBZ, $10 \mu \mathrm{l}$ of acetic acid, and $0.5 \mathrm{~g}$ of a $4-\AA$ molecular sieve, at $50^{\circ} \mathrm{C}$ for $3 \mathrm{~h}$. After the reaction, the nanosheets were sonicated in methanol for $1 \mathrm{~min}$, washed with methanol and subsequently dried in vacuum. The reacted NBZ on the nanosheets was collected by immersing the nanosheets in $4 \mathrm{ml}$ of water that contained $50 \mu \mathrm{l}$ of acetic acid. The concentration of reacted NBZ was measured by using a UV-vis spectrophotometer. A standard curve was generated by preparing various concentrations of NBZ and plotted against the NBZ concentration. The calculation was based on the equation $A=\varepsilon b c$, where $A$ is the absorbance (optical density) of solution in the transparent cell, $\varepsilon$ is the 
molar absorptivity $\left(0.015 \mathrm{~cm}^{-1} \mu \mathrm{M}^{-1}\right), b$ is the transparent cell path length $(1 \mathrm{~cm})$ and $c$ is the NBZ concentration. The surface concentration was calculated by using $c \times N_{\mathrm{A}} / S$, where $N_{\mathrm{A}}$ is Avogadro's number $\left(6.02 \times 10^{23}\right)$, and $S$ is the sample surface area $\left(1.76 \mathrm{~cm}^{2}\right)$.

The tested therapeutic gene was the plasmid DNA for GATA-binding protein 4 (GATA4), which is an early cardiac transcription factor. To generate the construct, the human GATA4 was amplified by using the polymerase chain reaction (PCR) and GATA4 primers. The procedures were confirmed by agarose gel electrophoresis. The GATA4 fragment was cloned into the pTracerCMV/Bsd vector (Life Technologies, Grand Island, NY, USA). Escherichia coli were used to amplify the plasmids. The plasmids were purified with a plasmid purification kit and verified by agarose gel electrophoresis. The GATA4 plasmid-encoding GFP fluorescence that was constructed for this study is shown in Figure 2b.

Preliminary experiments were performed on HEK cells (Supplementary Methods). For the main study, human MSCs derived from umbilical cord (hMSCs, the first passage; BIONET, Taiwan) were used. The genuineness of hMSCs was verified by using flow cytometry (Supplementary Figure S1). hMSCs were cultured in an alpha minimum essential medium with the standard cell culture protocol. The cells of passages between 6 and 8 were used. To transfect the cells, a new protocol was developed. The cells were cultured in 24-well tissue culture plates (tissue culture polystyrene; TCPS) or those containing planar silica ('Planar'), non-modified silica upright nanosheets ('Nano'), and 3-aminopropyltriethoxysilane-modified silica upright nanosheets ('Nano- $\mathrm{NH}_{2}$ '), where they were concurrently exposed to the naked GATA4 plasmids. The cells were seeded at a concentration of $5 \times 10^{4}$ cells in $1 \mathrm{ml}$ of serum-free medium with (or without) $1 \mu \mathrm{g}$ of plasmid. After $12 \mathrm{~h}$, the medium was changed to $10 \%$ serum-containing medium without any plasmid. After $48 \mathrm{~h}$ (at $60 \mathrm{~h}$ post cell seeding), the cells were harvested with $0.25 \%$ trypsin. For the PolyFect-transfected group (conventional control), the cells were transfected with the plasmid/PolyFect complexes by following the manufacturer's protocol. The cells were seeded $\left(5 \times 10^{4}\right.$ cells ml $\left.{ }^{-1}\right)$ on Planar in a $10 \%$ serum-containing medium for $12 \mathrm{~h}$. The medium was changed to a $10 \%$ serum-containing medium with $1 \mu \mathrm{g}$ of plasmid DNA and $3 \mu \mathrm{g}$ of PolyFect (Qiagen, Hilden,

a
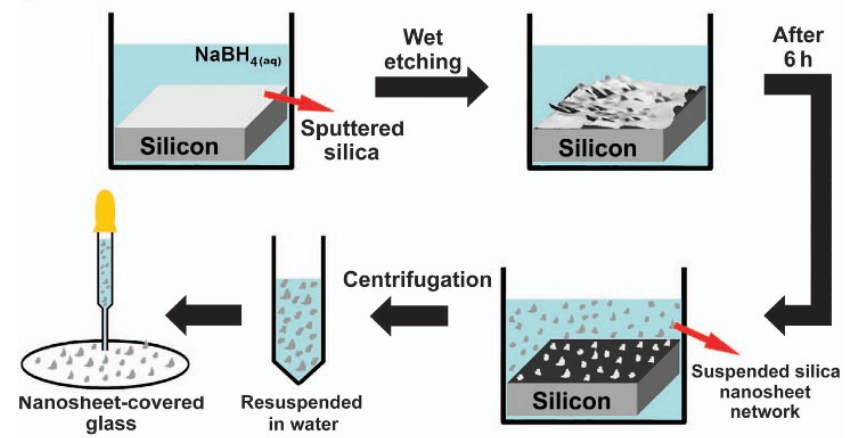

b
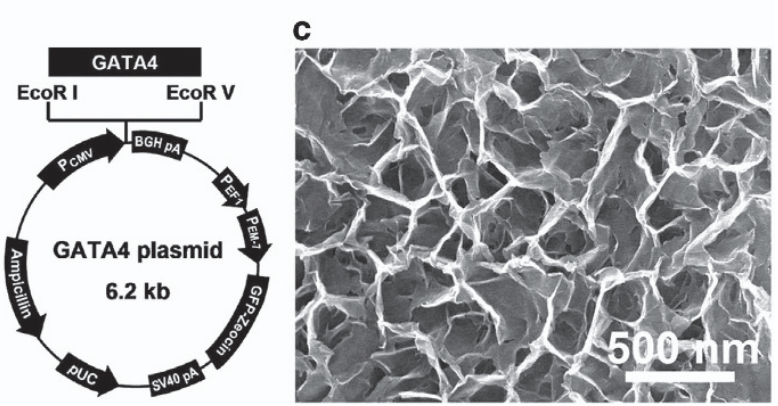

Figure 2 (a) Preparation process of the silica upright nanosheet network that was coated on glass. (b) Structure map of the green fluorescent proteinencoded GATA4 plasmid DNA. (c) Scanning electron microscopy image of the silica upright nanosheet network.
Germany; a dendrimer-based transfection reagent). After $24 \mathrm{~h}$ (at $36 \mathrm{~h}$ post cell seeding), the medium was changed to a $10 \%$ serum-containing medium without the plasmid. After another $48 \mathrm{~h}$ (at $84 \mathrm{~h}$ post cell seeding), the cells were harvested using a treatment with trypsin. In all groups, the expression of green fluorescence was observed using a confocal microscope (Pathway 435 BioImager, BD Biosciences, San Jose, CA, USA). The plasmid-treated cells were replated on TCPS, and the nuclei were stained with 4', 6'-diamidino-2phenylindole (DAPI). To determine the cell survival, the cell pellets were resuspended in phosphate-buffered saline with propidium iodide. A flow cytometer (FACS Caliber, BD Biosciences) was used to obtain the transfection efficiency and cell survival rate. Real-time reverse transcription-PCR (RT-PCR) was used to verify the gene expression of GATA4 and the other two cardiacassociated genes (myocyte enhancer factor 2C (MEF2C) and T-box 5 (TBX5)) at 60 or $84 \mathrm{~h}$. The gene expressions of integrins, FAK, RhoA and Racl were analyzed using RT-PCR at 12 and $60 \mathrm{~h}$ after cell seeding (details in Supplementary Table S1 and Supplementary Methods). For immunostaining, the cells were fixed with $4 \%$ paraformaldehyde for $30 \mathrm{~min}$, permeabilized with $0.5 \%$ Triton X-100 and $10 \%$ bovine serum albumin in phosphate-buffered saline for $1 \mathrm{~h}$ and subsequently immunostained with primary antibodies for the cardiac-associated maker proteins GATA4, NK-2 transcription factor related, locus 5 (NKX2.5), myosin-heavy chain 6 (MYH6) and ZO-1 (Santa Cruz Biotechnology, Dallas, TX, USA) at $4{ }^{\circ} \mathrm{C}$ for $24 \mathrm{~h}$ and the appropriate secondary antibodies at $4^{\circ} \mathrm{C}$ for $24 \mathrm{~h}$. After phosphate-buffered saline washing, the cells were stained with DAPI and observed with the confocal microscope.

\section{RESULTS AND DISCUSSION}

The silica upright nanosheet network, which was optimized for gene delivery, had a pore diameter of $400 \mathrm{~nm}$, an upright wall thickness of $5 \mathrm{~nm}$ and a wall height of $120 \mathrm{~nm}$ on an average, as shown in Figure 2c. The surface contact angle and surface zeta potential are shown in Table 1 . The contact angle of all surfaces was $\sim 50^{\circ}$. The surface zeta potential of the Planar and Nano was slightly negative $(-3 \mathrm{mV})$, whereas that of $\mathrm{Nano}-\mathrm{NH}_{2}$ was positive $(5-6 \mathrm{mV})$. The attenuated total reflectance-infrared spectroscopy spectra demonstrated the peak of $-\mathrm{NH}_{2}$ at $\sim 1570 \mathrm{~cm}^{-1}$ on the ATPES-modified nanosheets (Supplementary Figure S2). The energy dispersive X-ray spectrometer data are shown in Table 2. The elemental analysis revealed the existence of nitrogen and a reduced silicon content on the ATPES-modified nanosheets. Surface functionalization by amino groups was also quantified via a reaction with NBZ. The density of the amine groups that were exposed to the surface was $\sim 31.9$ molecules per $\mathrm{nm}^{2}$.

Table 1 Contact angle and zeta potential of planar silica (Planar), pristine nanosheets (Nano) and modified nanosheets ( $\mathrm{Nano}-\mathrm{NH}_{2}$ )

\begin{tabular}{lcc} 
& Contact angle $\left(^{\circ}\right)$ & Zeta potential (mV) \\
\hline Planar & 47.2 & -3.21 \\
Nano & 50.0 & -3.47 \\
Nano- $\mathrm{NH}_{2}$ & 49.9 & 5.45 \\
\hline
\end{tabular}

Table 2 Surface element analysis for the silica nanosheets

\begin{tabular}{lllc}
\hline Substrate atomic & Si (\%) & O (\%) & N (\%) \\
\hline Planar & 26.35 & 73.66 & - \\
Nano & 23.67 & 76.97 & - \\
Nano-NH & 16.57 & 68.57 & 14.86 \\
\hline
\end{tabular}


A preliminary test was performed to show that the naked plasmid could be successfully delivered into HEK cells on the nanosheets (Supplementary Figure S3). The green fluorescence (GFP) expression for transfected cells on Planar, Nano and $\mathrm{Nano}-\mathrm{NH}_{2}$, compared with the conventional PolyFect-transfected group, is demonstrated in Figure 3a. The transfection efficiency (percent cells with green fluorescence), which was estimated from the image analysis and quantified by using flow cytometry, is shown in Figures $3 \mathrm{~b}$ and $\mathrm{c}$. The cell survival rate is shown in Figure $3 \mathrm{~d}$. These data reveal that the transfection efficiency was much greater for the cells plated on Nano $(\sim 75 \%)$ and Nano- $\mathrm{NH}_{2}(\sim 85 \%)$ vs those on Planar $(<20 \%)$. Moreover, the transfected cells on Planar showed only weak fluorescence under the confocal microscope. Although the efficiency of the conventional PolyFect transfection was close to that of the vectorfree upright nanosheet groups, the cell survival rate in the PolyFect- transfected group was only $\sim 3 \%$, which was significantly lower than that observed in any of the vector-free groups ( $80-90 \%$ survival rate). Considering both efficiency and survival rate, 70 out of 100 starting cells were successfully transfected by the nanosheet approach vs three cells by the conventional approach.

RT-PCR was used to verify the gene expression of GATA4, and the results are shown in Figure 4a. The control hMSCs (without plasmid treatment) showed very-low levels of GATA4 gene expression on all three silica surfaces (Planar, Nano and Nano- $\mathrm{NH}_{2}$ ) and TCPS. The naked plasmid-exposed hMSCs on Nano- $\mathrm{NH}_{2}$ and Nano showed greater expression of GATA4 than those on Planar, TCPS, or any of the untreated controls. In particular, the gene expression of GATA4 for cells on Nano- $\mathrm{NH}_{2}$ and Nano reached a similar level as that of the PolyFect-transfected group. The plasmid-exposed hMSCs on TCPS showed no difference from any of the untreated controls, whereas a
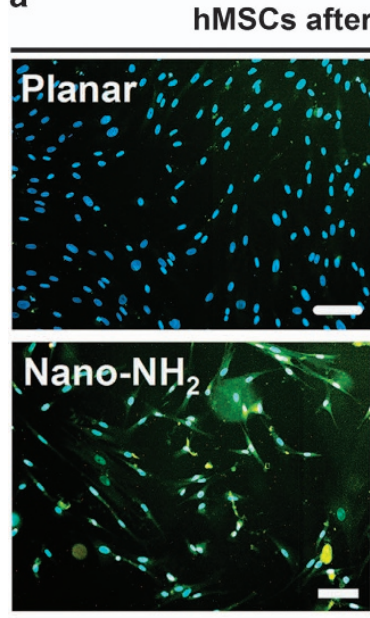

C

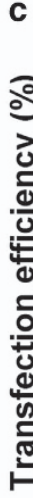

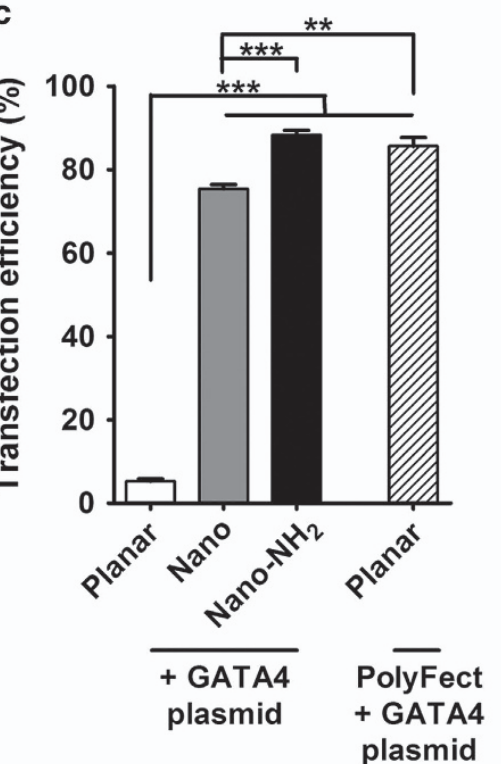

b

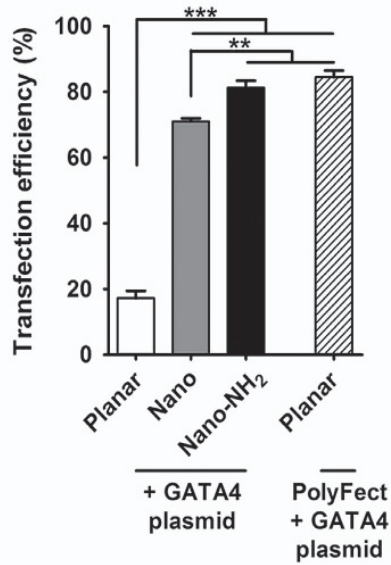

d

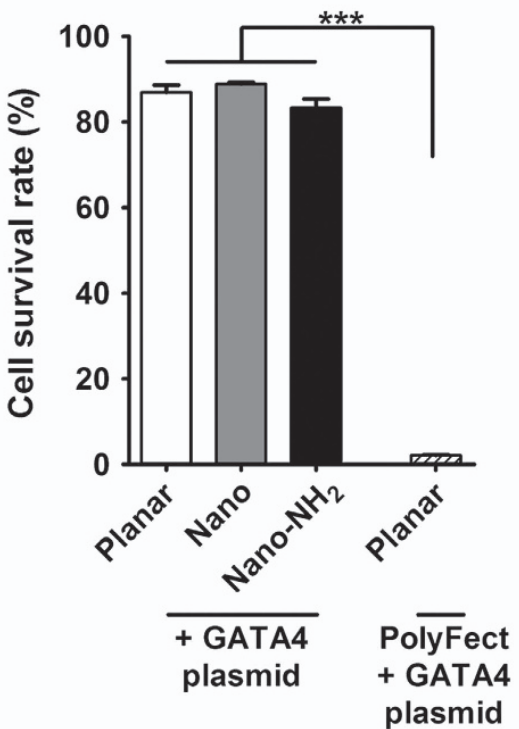

Figure 3 (a) Fluorescent images of human-umbilical-cord-derived mesenchymal stem cells, which were transfected with GATA4 plasmid on a planar silica surface (Planar), non-modified nanosheets (Nano), amino-modified nanosheets ( $\mathrm{Nano}_{\mathrm{N}} \mathrm{NH}_{2}$ ) and the conventional PolyFect-transfected group (on the Planar surface). The scale bar represents $50 \mu \mathrm{m}$. (b, c) Transfectability of human-umbilical-cord-derived mesenchymal stem cells expressed as the estimated percentage of total cells from the image analysis (b) and the gene transfection efficiency, which was precisely quantified using traditional flow cytometry (c). (d) Cell survival rate of human-umbilical-cord-derived mesenchymal stem cells, which was determined using flow cytometry. All cells were analyzed at $60 \mathrm{~h}$ post seeding except the PolyFect group (at $84 \mathrm{~h}$ ). Multiple samples were used in each independent experiment. The reproducibility was confirmed in at least three independent experiments. The statistical significance difference was evaluated by using one-way analysis of variance. ${ }^{* *} P<0.01 ; * * * P 0.001$. 
a

GATA4
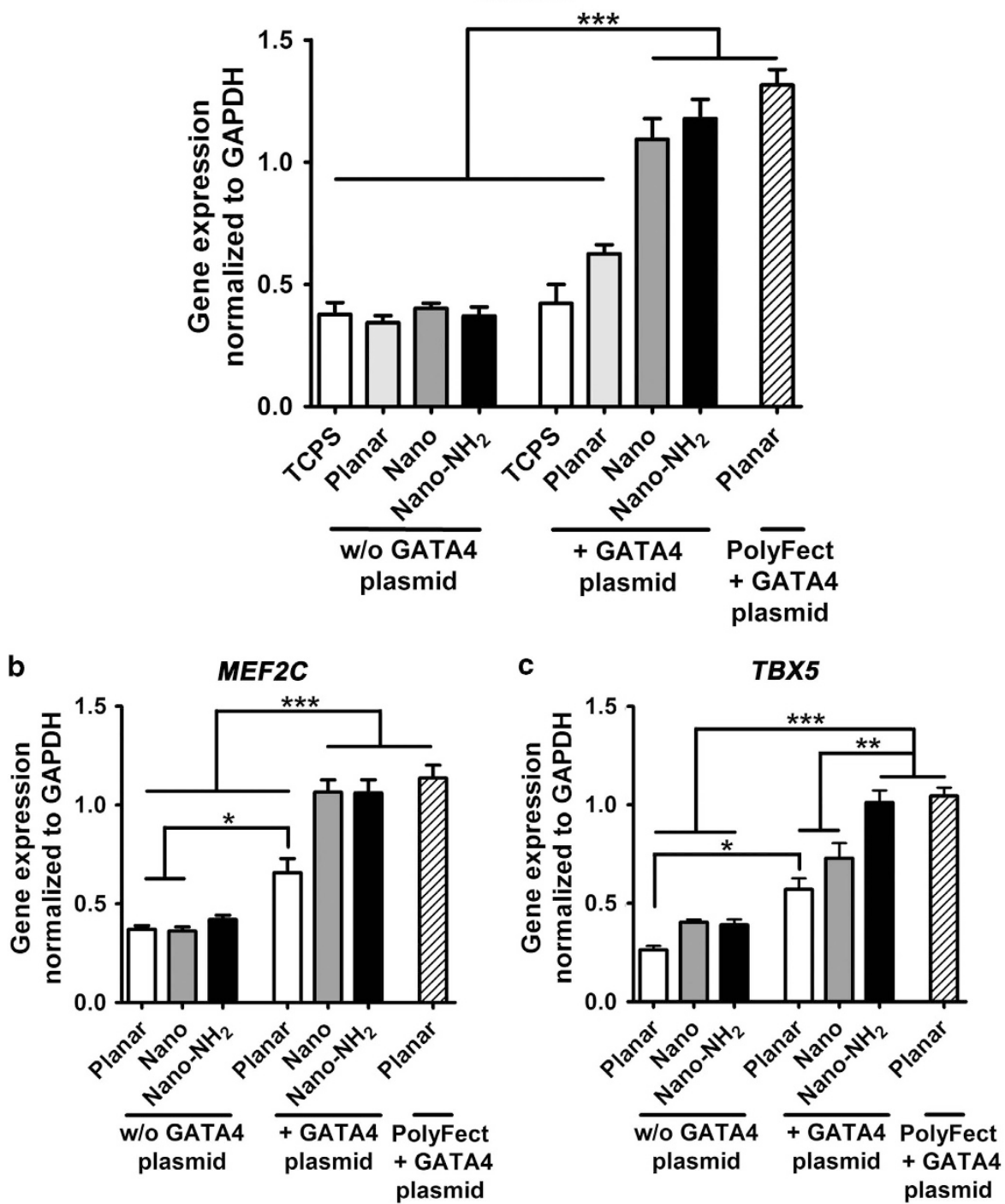

Figure 4 (a) Transfectability of human-umbilical-cord-derived mesenchymal stem cells, which was expressed as the level of GATA4 gene expression on the planar silica surface (Planar), non-modified nanosheets (Nano) and amino-modified nanosheets $\left(\mathrm{Nano}_{\mathrm{N}} \mathrm{NH}_{2}\right)$ in comparison to the regular tissue culture polystyrene plate. The expression levels were normalized to the housekeeping gene (GAPDH). ${ }^{*} P<0.05 ;{ }^{*} P<0.01$; ${ }^{* *} P<0.001$. (b, c) Expression of the other two cardiac-associated genes (MEF2C and TBX5) for GATA4-transfected human-umbilical-cord-derived mesenchymal stem cells on various substrates. ${ }^{*} P<0.05 ;{ }^{* *} P<0.01 ;{ }^{* *} P<0.001$.

those on Planar showed a slight increase but did not achieve statistical significance. When the plasmid loading was increased to $10 \mu \mathrm{g} \mathrm{ml}^{-1}$, the GATA gene expression was enhanced by 10 -fold over the control (Supplementary Figure S4). However, only $1 \mu \mathrm{g} \mathrm{ml} l^{-1}$ of plasmid was sufficient to induce protein expression, as will be described later. We also examined the delivery efficiency of GFP-encoded siRNA on the nanosheets (Supplementary Figure S5). The intracellular delivery of siRNA on Nano and Nano- $\mathrm{NH}_{2}$ was enhanced over the control by approximately seven- and ninefold, respectively. The delivery efficiency $(55-65 \%)$ was at a similar level to that of PolyFect ( 58\%). These results confirm that the nanosheet platform can also be used to enhance the delivery efficiency of siRNA.

To consolidate the effect of GATA4 gene delivery on cells, the other two cardiac-associated genes (MEF2C and TBX5) were further analyzed. The control hMSCs without the plasmid treatment showed low expressions of MEF2C and TBX5 on all surfaces (Figures 4b and c).
The plasmid-treated hMSCs on Nano- $\mathrm{NH}_{2}$ showed greater expression of MEF2C and TBX5 than those on Planar or any of the untreated controls. On Nano and Nano- $\mathrm{NH}_{2}$, the extent of increase in both GATA4 and MEF2C expression in transfected cells was both similar to that in the PolyFect-transfected group. However, the expression of TBX5 was significantly upregulated only for the cells on $\mathrm{Nano}-\mathrm{NH}_{2}$ and for the cells transfected by PolyFect. The above effect was specific to hMSCs. Upregulation of the MEF2C and TBX5 genes was not observed in GATA4-transfected HEK cells (Supplementary Figure S3).

The images of immunofluorescence staining for cardiac-associated marker proteins are displayed in Figure 5. The expressions of GATA4, NKX2.5, MYH6 and ZO-1 were positive for the cells plated on Nano $(\sim 70 \%)$ and $\mathrm{Nano}^{-\mathrm{NH}_{2}}(\sim 80 \%)$, which approached those in the PolyFect group ( $\sim 90 \%$, quantified in Supplementary Figure S6). On Planar, only GATA4 showed a weak intensity $(\sim 3 \%)$. No expression was observed in any nontransfected group. 


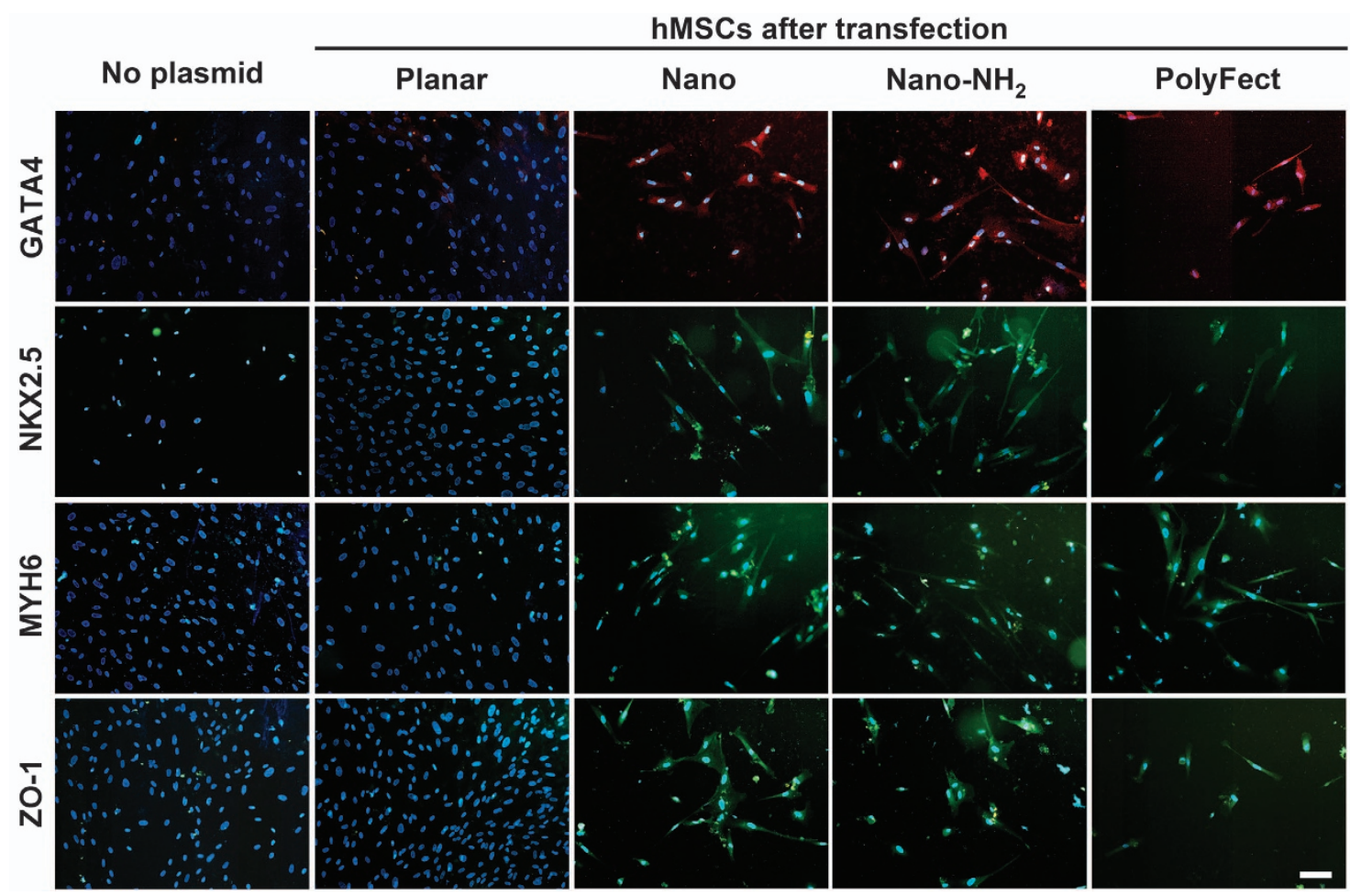

Figure 5 Immunofluorescence staining of human-umbilical-cord-derived mesenchymal stem cells transfected with GATA4 plasmid on a planar silica surface (Planar), non-modified nanosheets (Nano) and amino-modified nanosheets (Nano- $\mathrm{NH}_{2}$ ), the conventional PolyFect-transfected group (on Planar) and non-transfected control (on Planar) after 7 days. Scale bar: $50 \mu \mathrm{m}$.

Previous literature has explored the surface designs of gene vectors or NPs to improve gene delivery. The positive charge of a gene carrier often facilitates the cellular entry and hence the gene delivery efficiency. Examples include boron nitride nanotubes with surfaces modified by 3-aminopropyltriethoxysilane-mesoporous silica, ${ }^{18}$ amino-modified silica NPs ${ }^{19}$ and so on. However, in most situations, the positively charged gene carrier suffers from cytotoxicity. ${ }^{20}$ In our study, the 3-aminopropyltriethoxysilane-modified nanosheets and pristine nanosheets had similar transfection efficiencies and cell survival rates. This finding suggests that the positive charge on silica nanosheets and the electronic interaction between nanosheets and seeded cells may not be the dominating mechanisms behind the enhanced gene delivery observed in this study.

Gene delivery may also be facilitated by the surface properties of a culture substrate, such as hydrophobicity. ${ }^{21}$ However, our silica upright nanosheets have rather good wetting properties (contact angle $\sim 50^{\circ}$ ). Because the planar silica had a weaker effect on transfection than TCPS, the silica material may play a role in the nanosheet-induced gene transfection. Nanotopography, such as nanoscale surface roughness on NPs, is considered to promote gene delivery. Nanoporous silica NPs (but not the nonporous ones) increase the transfection. ${ }^{22}$ Silica NPs decorated with $\sim 300$ spikes $\sim 6.4 \mathrm{~nm}$ in diameter to mimic the enveloped virus can enhance gene binding and delivery, although they have higher cytotoxicity. ${ }^{23}$ Based on these arguments, the surface structures or textures, particularly in the form of spikes or upright nanosheets, may promote gene delivery. In particular, our upright nanosheets and amino-modified nanosheets promote gene delivery as effectively as the rough NPs in the literature ${ }^{23}$ without sacrificing the cell viability. In the literature, the intracellular delivery of plasmids using nanopillars (200 nm diameter) requires a cytotoxic transfection reagent (Lipofectamine). ${ }^{7}$ Nanowires that can pierce into cells are rather cytotoxic and not as effective in the naked DNA delivery. ${ }^{8,9}$ The high cell survival rate on our silica upright nanosheets suggests that the nanosheets may not penetrate the cell membrane, which is similar to the case of nanopillars. ${ }^{24}$ Regarding the size of the nanosheet features (thickness and depth), we could control the procedures, such as the etching time, to obtain nanosheets with different geometric parameters, for example, those with a wall thickness of $5-400 \mathrm{~nm}$ and a height of $50-1100 \mathrm{~nm}$. A series of experiments suggested that the aspect ratio appeared to be the critical parameter, and when the aspect ratio was less than 5 , the transfection efficiency was lower than $60 \%$. More efforts are required to identify the relative importance of each factor, which will be a subject of future studies.

The existing mechanisms fail to account for the remarkable substrate-dictating transfection of naked DNA on the silica upright nanosheets. Moreover, surface nanotopography may activate integrins and FAK by the cell adhesion-mediated signaling mechanism. ${ }^{25}$ Thus, we attempted to interpret the new findings based on how surface nanotopography might affect the cellular function (integrins/FAK/ cytoskeleton) and consequently the transfectability.

Integrins can activate the intracellular molecules FAK and the two small GTPases that regulate intracellular dynamics (RhoA and Rac1). The FAK molecule is a central modulator of cytoskeleton arrangement, and its expression is affected by material morphology. ${ }^{26}$ FAK can activate RhoA and Racl, whereas the upregulation of RhoA and Rac1 promotes gene delivery. ${ }^{27,28}$ RhoA controls the formation of stress fibers, and Racl induces the formation of lamellipodia. Both RhoA and Racl induce the organization of actin filaments and regulate cell morphology, cell cycle progression and gene expression. ${ }^{29}$ The gene expressions of integrins, FAK, RhoA and Racl, analyzed using RT-PCR at 12 and $60 \mathrm{~h}$ after cell seeding, are shown in 
a

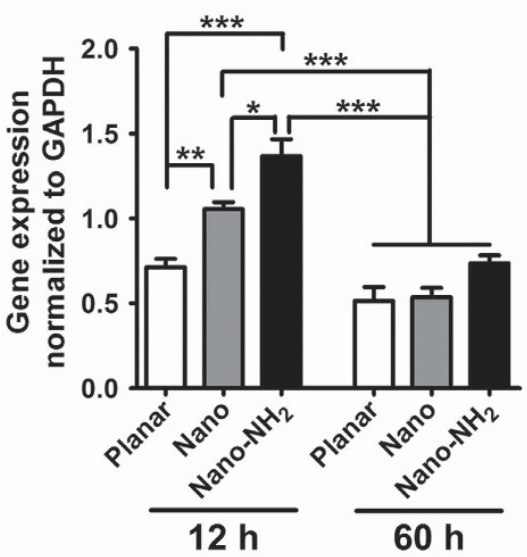

d

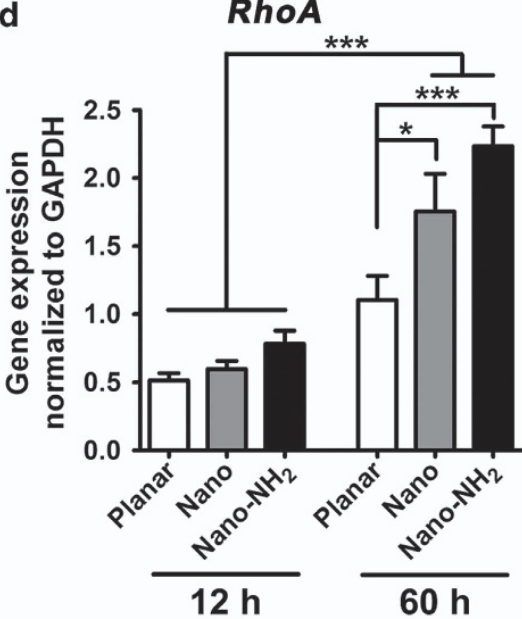

b

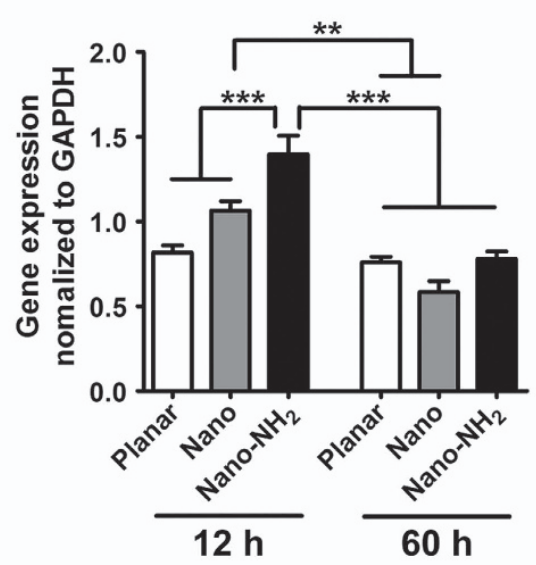

e

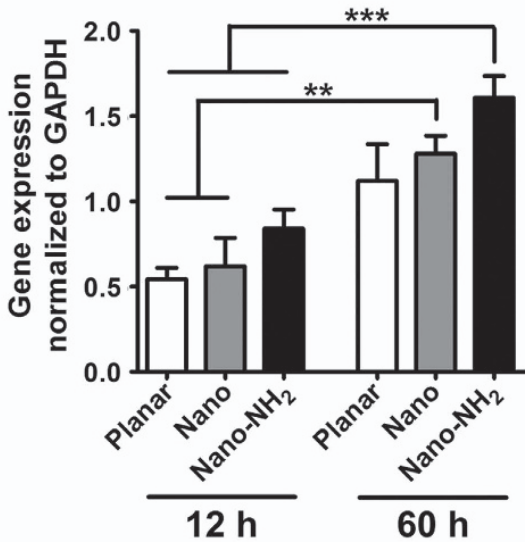

C

FAK

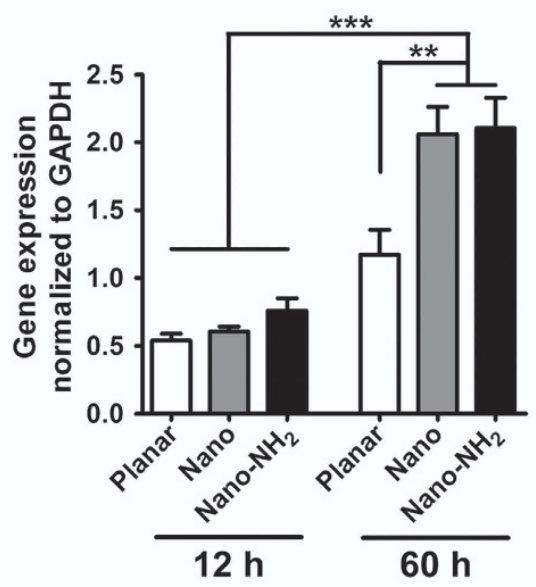

f

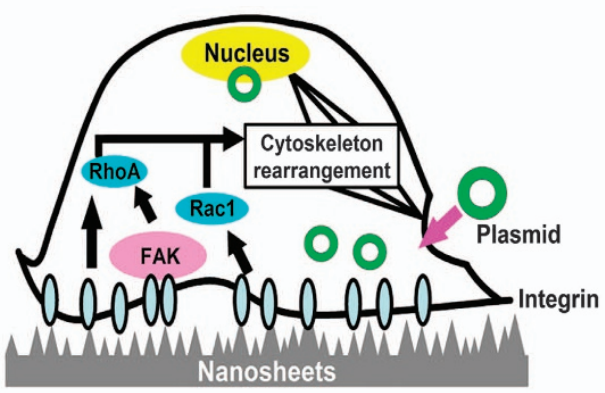

Figure 6 Expression of integrin and the intracellular molecule genes at $12 \mathrm{~h}$ and $60 \mathrm{~h}$ post cell seeding, including that of (a) ITG $\beta 3$, (b) ITG $\alpha V$, (c) focal adhesion kinase (FAK), (d) RhoA and (e) Racl, on the planar silica surface (Planar), non-modified nanosheets (Nano) and amino-modified nanosheets (Nano$\mathrm{NH}_{2}$ ). ${ }^{*} P<0.05 ;{ }^{* *} P<0.01 ;{ }^{* *} P<0.001$. (f) A hypothetical mechanism behind the nanosheet-induced signal transduction and gene delivery.

Figures $6 \mathrm{a}$ and e. The expressions of integrin $\beta 3$ (ITG $\beta 3$ ) and integrin $\alpha \mathrm{v}$ (ITG $\alpha v)$ revealed similar tendencies of change. These two genes were quickly upregulated for the cells on $\mathrm{Nano}-\mathrm{NH}_{2}$ at $12 \mathrm{~h}$, which did not endure at $60 \mathrm{~h}$. The gene regulation of ITG $\beta 3$ and ITG $\alpha v$ was also observed on Nano at $12 \mathrm{~h}$, but ITG $\alpha v$ had no statistically significant difference from that on the Planar group. Although the other integrin $\beta 1$ (ITG $\beta 1$ ) is linked to the cytoskeleton on nanomaterials ${ }^{30,31}$ and integrin $\beta 8$ (ITG $\beta 8$ ) is associated with ITG $\alpha v^{32}$, we did not observe any significant difference in these integrins among the substrates (Supplementary Figure S7). However, we noted that the involved integrins may be different for different cell types (Supplementary Figure S8). Genetic modulations of intracellular molecules FAK, RhoA and Racl were also observed on the nanosheets. The expressions of FAK, RhoA and Racl at $12 \mathrm{~h}$ were at similar levels on Planar, Nano and Nano- $\mathrm{NH}_{2}$. After $60 \mathrm{~h}$, the expressions of FAK and RhoA were upregulated for the cells on Nano and $\mathrm{Nano}-\mathrm{NH}_{2}$ (Figures $6 \mathrm{c}$ and e). Therefore, we hypothesize that the upright nanosheets may promote the delivery of naked plasmid (in this case, GATA4) by enhancing the expression of integrins on the cell membrane. Then, the integrins may activate the intracellular molecules FAK, RhoA and Racl to affect the cytoskeleton arrangement, which increases the plasmid uptake and later transfer to the nuclei (Figure 6f).

A few other mechanisms have been proposed to increase transfection in the literature, such as passive delivery by concentration difference, ${ }^{33}$ direct penetration of nanowires ${ }^{8,9}$ and mechanical stimulation by microfluidics, which creates transient holes on the cell membrane that enable the diffusion of biomolecules into the cells. ${ }^{34}$ Unlike these previous approaches, the plasmids in our study were not packaged or pre-adsorbed on a material surface before cell seeding. Instead, the cells and plasmids were plated at the same time ('concurrent' transfection) on the nanosheets to improve the transfection efficiency. The high cell survival rate on our silica upright nanosheets suggests that the nanosheets may not pierce into the cells. The characteristic size of the nanosheets may also be too small to generate compression or shear forces for cell squeezing. Moreover, a recent theoretical article showed that the time for plasmid DNA to directly pass through the small pores on the plasma membrane of the cells was much longer. ${ }^{35}$ Therefore, the delivery mechanism by the nanosheets may be quite different from those described in prior literature. 
The material surface can adsorb serum proteins (for example, vitronectin, fibronectin and so on) that bind with the integrin receptors (for example, integrin $\alpha v \beta 3$ ), which affects their expression on the cell membrane of human MSCs. ${ }^{36}$ Because we did not use serum during transfection, the material surface appeared to directly interact with integrins. The upright wall of the silica nanosheets had a thickness close to the diameter $(8-12 \mathrm{~nm})$ of the integrin $\alpha v \beta 3$ molecules. ${ }^{25}$ Moreover, the 'continuous' network (upright wall) in the current study, instead of the more commonly reported discrete nanofeatures (for example, nanoislands), may induce integrin clustering, which is critical for integrin activation. ${ }^{37}$ Therefore, we hypothesize that the interaction between the nanosheets and the cells may activate the integrin $\alpha v \beta 3$ receptor of MSCs. Nevertheless, integrin activation by the upright nanosheets appeared transient. The gene expression of integrin $\alpha v \beta 3$ was downregulated at $60 \mathrm{~h}$. The timedependent regulation of integrins/FAK suggests that the delivery of naked GATA4 plasmid may be facilitated through the changes in the cell surface receptor and cytoskeleton on the nanosheets in a timely fashion. We observed that if the plasmids were added at $12 \mathrm{~h}$ after cell seeding, the transfectability on Nano- $\mathrm{NH}_{2}$ was modestly decreased. If the plasmids were added at $36 \mathrm{~h}$ after cell seeding, the transfectability on Nano- $\mathrm{NH}_{2}$ was significantly reduced (Supplementary Figure S9). However, if the plasmids were added at $12 \mathrm{~h}$ before cell seeding, the transfectability on Nano- $\mathrm{NH}_{2}$ was slightly increased. These data suggested that the increased gene entry may have a limited time frame ('transfection window'). This suggestion was conceivable based on the cytoskeleton hypothesis. If the plasmids are exposed too late, the transient cellular machine may cease to function before they are transported from the cytosol to the nucleus, which decreases the transfection efficiency.

Regarding the benefit of GATA4 plasmid delivery to stem cells, GATA4 is an important transcription factor for cardiac development in embryos and has been used as a therapeutic gene for treating cardiac diseases. ${ }^{38,39}$ Fibroblasts transduced by the three genes GATA4, $M E F 2 C$, and TBX5 ('GMT') can be reprogrammed into cardiomyocytes that repair the myocardial heart when injected. ${ }^{39}$ GATA4 is upstream of MEF2 and TBX5, which interact with GATA4. ${ }^{38}$ In our study, GATA4, MEF2C and TBX5 were upregulated for MSCs transfected with the naked GATA4 plasmid, suggesting dynamic interaction of these genes in stem cells. In contrast, the $M E F 2 C$ and TBX5 gene expression levels were not upregulated for the HEK cells transfected with GATA4. The cytoskeleton change of MSCs on the nanosheets did not impair the stemness of MSCs (Supplementary Figure S10). The synergistic effects of cardiac-associated markers in MSCs, but not HEK, may further contribute to the cardiogenesis of the stem cells on glass nanosheets. Indeed, MSCs express cardiac marker proteins after GATA transfection. This result suggests that MSCs may be effectively reprogrammed by the nanosheet-induced GATA4 transfection, which deserves further investigation. The increase in gene expression for GATA4-transfected cells was only two- to threefold while cells could express specific marker proteins after 7 days. This may be related to the dynamic interaction of the GMT genes. The expression of marker proteins at 7 days further suggested that the effect of the new transfection may not be transient only. In view of these points, the current platform is superior to other systems regarding the shorter time ( 7 days vs $2-4$ weeks) in inducing cell differentiation.

Finally, the dimensions of the nanosheet network used in this study were optimized. The height and thickness ratio of the nanosheets appears to be the important factor that affects the transfection efficiency of the nanosheets. A mechanistic study on various nanosheets may help clarify the exact relation between geometric parameters and integrin activation. Moreover, the surface rigidity or chemistry of the nanosheet may have a role in gene transfection, which may be verified by surface modification with different compounds or ligands. Because the nanosheets are thin and may be deposited on different substrates, they may be further developed as an in vivo platform for temporo-spatially controllable gene delivery in the future.

\section{CONCLUSION}

The silica upright nanosheets can promote the transfection of naked plasmid DNA (GATA4) into human MSCs or HEK cells without any transfection reagent or NP vector. The vector-free delivery of naked plasmid is achieved by the integrin-FAK-Rho signaling axis, which can be activated by nanotopography in a limited time frame. The technology is more cell number-effective $(70 \%$ of starting cells successfully transfected and alive), more time-efficient ( $24 \mathrm{~h}$ shorter), and much less cytotoxic than the conventional transfection reagent. Transfecting GATA4 plasmids into human-umbilical-cord-derived MSCs can upregulate the other two important cardiac marker genes (MEF2C and TBX5). In addition to greater viability, MSCs express cardiac marker proteins at 7 days following transfection. Particularly, the high-yield preparation of a transfection dish from abundant materials (silica glass) with a very simple method (wet etching/heating) without additional surface modification will be very attractive and available even in developing countries and under limited conditions. Future efforts include fine-tuning the geometrical scales of the upright nanosheets to identify the critical parameters (for example, sheet thickness and height) to evaluate the effect on inducing stem cell differentiation. The innovative technology for the intracellular delivery of therapeutic genes through silica upright nanosheets and its possibility to reprogram the cells may have potential applications in gene- and cell-based medicines.

\section{CONFLICT OF INTEREST}

The authors declare no conflict of interest.

\section{ACKNOWLEDGEMENTS}

This research was supported by the Ministry of Education Taiwan through an international excellence NTU-NIMS collaboration grant (grant no.

102R104100) and Ministry of Science Technology Taiwan (MOST103-2321B-002-100). The MSCs of the first passage were supplied by Dr M Sieber of BIONET Taiwan.

1 Mintzer, M. A. \& Simanek, E. E. Nonviral vectors for gene delivery. Chem. Rev. 109 259-302 (2009).

2 Wu, Y.-L., Putcha, N., Ng, K. W., Leong, D. T., Lim, C. T., Loo, S. C. J. \& Chen, X Biophysical responses upon the interaction of nanomaterials with cellular interfaces. Acc. Chem. Res. 46, 782-791 (2013).

3 Tay, C. Y., Cai, P., Setyawati, M. I., Fang, W., Tan, L. P., Hong, C. H. L., Chen, X. \& Leong, D. T. Nanoparticles strengthen intracellular tension and retard cellular migration. Nano Lett. 14, 83-88 (2014).

4 Mehier-Humbert, S. \& Guy, R. H. Physical methods for gene transfer: improving the kinetics of gene delivery into cells. Adv. Drug Deliv. Rev. 57, 733-753 (2005)

5 Tang, L., Liu, W. \& Liu, G. High-strength hydrogels with integrated functions of $\mathrm{H}$-bonding and thermoresponsive surface-mediated reverse transfection and cell detachment. Adv. Mater. 22, 2652-2656 (2010).

6 Li, K., Feng, L., Shen, J., Zhang, Q., Liu, Z., Lee, S.-T. \& Liu, J. Patterned substrates of nano-graphene oxide mediating highly localized and efficient gene delivery. ACS Appl. Mater. Interfaces 6, 5900-5907 (2014).

7 Teo, B. K. K., Goh, S. H., Kustandi, T. S., Loh, W. W., Low, H. Y. \& Yim, E. K. F. The effect of micro and nanotopography on endocytosis in drug and gene delivery systems. Biomaterials 32, 9866-9875 (2011).

8 Kim, W., Ng, J. K., Kunitake, M. E., Conklin, B. R. \& Yang, P. D. Interfacing silicon nanowires with mammalian cells. J. Am. Chem. Soc. 129, 7228-7229 (2007). 
9 Shalek, A. K., Robinson, J. T., Karp, E. S., Lee, J. S., Ahn, D.-R., Yoon, M.-H., Suttona, A., Jorgollic, M., Gertnera, R. S., Gujrala, T. S., MacBeath, G., Yang, E. G. \& Park, H. Vertical silicon nanowires as a universal platform for delivering biomolecules into living cells. Proc. Natl Acad. Sci. USA 107, 1870-1875 (2010).

10 Adler, A. F. \& Leong, K. W. Emerging links between surface nanotechnology and endocytosis: impact on nonviral gene delivery. Nano Today 5, 553-569 (2010).

11 Bettinger, C. J., Langer, R. \& Borenstein, J. T. Engineering substrate topography at the micro- and nanoscale to control cell function. Angew. Chem. Int. Ed. Engl. 48, 5406-5415 (2009).

12 Geiger, B., Spatz, J. P. \& Bershadsky, A. D. Environmental sensing through focal adhesions. Nat. Rev. Mol. Cell Biol. 10, 21-33 (2009).

13 Dalby, M. J., Yarwood, S. J., Johnstone, H. J., Affrossman, S. \& Riehle, M. O. Fibroblast signaling events in response to nanotopography: a gene array study. IEEE Trans. Nanobiosci. 1, 12-17 (2002).

14 Teo, B. K. K., Wong, S. T., Lim, C. K., Kung, T. Y. S., Yap, C. H., Ramagopal, Y., Romer, L. H. \& Yim, E. K. F. Nanotopography modulates mechanotransduction of stem cells and induces differentiation through focal adhesion kinase. ACS Nano 7, 4785-4798 (2013).

15 Choia, C.-H., Hagvall, S. H., Wu, B. M., Dunn, J. C. Y., Beyguic, R. E. \& Kim, C.-J. Cell interaction with three-dimensional sharp-tip nanotopography. Biomaterials 28, 1672-1679 (2007).

16 Dalby, M. J., Gadegaard, N. \& Wilkinson, C. D. W. The response of fibroblasts to hexagonal nanotopography fabricated by electron beam lithography. J. Biomed. Mater. Res. Part A 84, 973-979 (2008).

17 Ji, Q., Yamazaki, T., Hanagata, N., Lee, M. V., Hill, J. P. \& Ariga, K. Silica-based gene reverse transfection: an upright nanosheet network for promoted DNA delivery to cells. Chem. Commun. 48, 8496-8498 (2012).

18 Li, X., Zhi, C., Hanagata, N., Yamaguchi, M., Bando, Y. \& Golberg, D. Boron nitride nanotubes functionalized with mesoporous silica for intracellular delivery of chemotherapy drugs. Chem. Commun. 49, 7337-7339 (2013).

19 Xiao, X., He, Q. \& Huang, K. Novel amino-modified silica nanoparticles as efficient vector for hepatocellular carcinoma gene therapy. Med. Oncol. 27, 1200-1207 (2010).

20 Nan, A., Bai, X., Son, S. J., Lee, S. B. \& Ghandehari, H. Cellular uptake and cytotoxicity of silica nanotubes. Nano Lett. 8, 2150-2154 (2008).

21 Shiu, J. Y., Kuo, C. W., Whang, W. T. \& Chen, P. L. Observation of enhanced cell adhesion and transfection efficiency on superhydrophobic surfaces. Lab Chip 10, 556-558 (2010).

22 Chen, Y., Chu, C., Zhou, Y., Ru, Y., Chen, H., Chen, F., He, Q., Zhang, Y., Zhang, L. \& Shi, J. Reversible pore-structure evolution in hollow silica nanocapsules: large pores for siRNA delivery and nanoparticle collecting. Small 7, 2935-2944 (2011).

23 Niu, Y., Yu, M., Hartono, S. B., Yang, J., Xu, H., Zhang, H., Zhang, J., Zou, J., Dexter, A., Gu, W. \& Yu, C. Nanoparticles mimicking viral surface topography for enhanced cellular delivery. Adv. Mater. 25, 1-5 (2013).

24 Hanson, L., Lin, Z. C., Xie, C., Cui, Y. \& Cui, B. Characterization of the cell-nanopillar interface by transmission electron microscopy. Nano Lett. 12, 5815-5820 (2012).

25 Arnold, M., Cavalcanti-Adam, E. A., Glass, R., Blümmel, J., Eck, W., Kantlehner, M., Kessler, H. \& Spatz, J. P. Activation of integrin function by nanopatterned adhesive interfaces. Chem. Phys. Chem. 5, 383-388 (2004).

26 Yim, E. K., Darling, E. M., Kulangara, K., Guilak, F. \& Leong, K. W. Nanotopographyinduced changes in focal adhesions, cytoskeletal organization, and mechanical properties of human mesenchymal stem cells. Biomaterials 31, 1299-1306 (2010).
27 Grassart, A., Meas-Yedid, V., Dufour, A., Olivo-Marin, J. C., Dautry-Varsat, A. \& Sauvonnet, N. Pak1 phosphorylation enhances cortactin-N-WASP interaction in clathrin-caveolin-independent endocytosis. Traffic 11, 1079-1091 (2010).

28 Lamaze, C., Dujeancourt, A., Baba, T., Lo, C. G., Benmerah, A. \& Dautry-Varsat, A. Interleukin 2 receptors and detergent-resistant membrane domains define a clathrinindependent endocytic pathway. Mol. Cell 7, 661-671 (2001).

29 Kerr, B. A., Otani, T., Koyama, E., Freeman, T. A. \& Enomoto-Iwamoto, M. Small GTPase protein Rac-1 is activated with maturation and regulates cell morphology and function in chondrocytes. Exp. Cell Res. 314, 1301-1312 (2008).

30 Boyd, N. D., Chan, B. M. C. \& Petersen, N. O. Adaptor protein-2 exhibits $\alpha 1 \beta 1$ or $\alpha 6 \beta 1$ integrin-dependent dedistribution in rhabdomyosarcoma cells. Biochemistry 41, 232-240 (2002)

31 Yang, K., Jung, K., Ko, E., Kim, J. i. n., Park, K. I., Kim, J. \& Cho, S.-W. Nanotopographical manipulation of focal adhesion formation for enhanced differentiation of human neural stem cells. ACS Appl. Mater. Interfaces 5, 10529-10540 (2013).

32 Barczyk, M., Carracedo, S. \& Gullberg, D. Integrins. Cell Tissue Res. 339, 269-280 (2010)

33 Singh, R. \& Lillard, J. W. Nanoparticle-based targeted drug delivery. Exp. Mol. Pathol. 86, 215-223 (2009).

34 Sharei, A., Zoldan, J., Adamo, A., Sim, W. Y., Cho, N., Jackson, E., Mao, S., Schneider, S., Han, M.-J., Lytton-Jean, A., Basto, P. A., Jhunjhunwala, S., Lee, J., Heller, D. A., Kang, J. W., Hartoularos, G. C., Kim, K.-S., Anderson, D. G., Langer, R. \& Jensen, K. F. A vector-free microfluidic platform for intracellular delivery. Proc. Natl Acad. Sci. USA 110, 2082-2087 (2013).

35 Budker, V., Budker, T., Zhang, G., Subbotin, V., Loomis, A. \& Wolff, J. A. Hypothesis: naked plasmid DNA is taken up by cells in vivo by a receptor-mediated process. J. Gene. Med. 2, 76-88 (2000)

36 Wiesner, S., Lange, A. \& Fassler, R. Local call: from integrins to actin assembly. Trends Cell Biol. 16, 327-329 (2006).

37 Huang, J. \& Ding, J. Nanostructured interfaces with RGD arrays to control cell-matrix interaction. Soft Matter 6, 3395-3401 (2010).

38 Houweling, A. C., Borren, M. M., Moorman, A. F. M. \& Christoffels, V. M. Expression and regulation of the atrial natriuretic factor encoding gene Nppa during development and disease. Cardiovasc. Res. 67, 583-593 (2005).

39 Qian, L., Huang, Y., Spencer, C. I., Foley, A., Vedantham, V., Liu, L., Conway, S. J., Fu, J.-d. \& Srivastava, D. In vivo reprogramming of murine cardiac fibroblasts into induced cardiomyocytes. Nature 485, 593-598 (2012).

(c) (i) This work is licensed under a Creative Commons Attribution 4.0 International License. The images or other third party material in this article are included in the article's Creative Commons license, unless indicated otherwise in the credit line; if the material is not included under the Creative Commons license, users will need to obtain permission from the license holder to reproduce the material. To view a copy of this license, visit http:// creativecommons.org/licenses/by/4.0/

Supplementary Information accompanies the paper on the NPG Asia Materials website (http://www.nature.com/am) 